\title{
${ }^{111}$ In-Octreotide Is Superior to ${ }^{123} \mathrm{I}$ - Metaiodobenzylguanidine for Scintigraphic Detection of Head and Neck Paragangliomas
}

\author{
Klaas Pieter Koopmans ${ }^{1}$, Pieter L. Jager ${ }^{1}$, Ido P. Kema ${ }^{2}$, Michiel N. Kerstens ${ }^{3}$, Frans Albers ${ }^{\dagger 4}$, and Robin P.F. Dullaart ${ }^{3}$ \\ ${ }^{I}$ Department of Nuclear Medicine and Molecular Imaging, University Medical Center Groningen, University of Groningen, Groningen, \\ The Netherlands; ${ }^{2}$ Department of Clinical Chemistry, University Medical Center Groningen, University of Groningen, Groningen, The \\ Netherlands; ${ }^{3}$ Department of Endocrinology, University Medical Center Groningen, University of Groningen, Groningen, The \\ Netherlands; and ${ }^{4}$ Department of Ear Nose and Throat Diseases, University Medical Center Groningen, University of Groningen, \\ Groningen, The Netherlands
}

\begin{abstract}
In this study, we evaluated the diagnostic yield of somatostatin receptor scintigraphy (SRS), I-metaiodobenzylguanidine (MIBG) scintigraphy, and morphologic imaging (CT or MRI) in patients with head and neck paragangliomas. Methods: In a university hospital setting, patients considered to have head and neck paraganglioma were referred to the outpatient endocrinology department and underwent CT or MRI, SRS, and MIBG imaging. For validation, we used a composite reference standard consisting of clinical and histologic data and CT or MRI, with which SRS and MIBG imaging were compared. Urinary metanephrine and normetanephrine measurements were also obtained. Results: Twenty-nine consecutively referred patients (17 women and 12 men) were included and were found to have paraganglioma. Both morphologic and SRS were positive in 27 patients (sensitivity, 93\%, and 95\% confidence interval [Cl], 77\%-98\%, compared with the composite reference standard), whereas MIBG was positive in only 13 patients $(44 \%$; $95 \% \mathrm{Cl}, 23 \%-61 \%)(P<0.001$, compared with SRS). On a lesion-based analysis, morphologic imaging detected 31 lesions (sensitivity, 82\%; 95\% Cl, 65\%92\%), SRS detected 34 (89\%; 95\% Cl, 75\%-97\%), and MIBG detected 15 (42\%; 95\% Cl, 26\%-59\%). SRS was superior to MIBG $(P=0.001)$. With SRS, a previously unknown carcinoid tumor was detected in 1 patient, and a carcinoid was suspected in another patient. MIBG detected an additional adrenal pheochromocytoma in 1 patient. Urinary metanephrine or normetanephrine excretion was elevated in 6 patients. The number of lesions on SRS and MIBG per patient correlated with the levels of abnormal metanephrine or normetanephrine excretion $(P=0.005$ and $P=0.02$, respectively). Conclusion: SRS was superior to MIBG in patients with highly suspected head and neck paraganglioma.
\end{abstract}

Key Words: endocrinology; oncology; neuroendocrine; accuracy; MIBG scintigraphy; octreotide scintigraphy; paraganglioma

J Nucl Med 2008; 49:1232-1237

DOI: 10.2967/jnumed.107.047738

\footnotetext{
Received Oct. 3, 2007; revision accepted Apr. 14, 2008.

For correspondence contact: Robin P.F. Dullaart, Department of Endocrinology, University Medical Centre Groningen, P.O. Box 30.001, 9700 RB Groningen, The Netherlands.

E-mail: r.p.f.dullaart@int.umcg.nl

${ }^{\dagger}$ Deceased.

COPYRIGHT @ 2008 by the Society of Nuclear Medicine, Inc.
}

$\mathbf{P}$ aragangliomas, rare tumors that originate from the paraganglia of the autonomous nervous system, constitute approximately $0.6 \%$ of head and neck tumors (1). These tumors most commonly are associated with the parasympathetic nervous system along the cranial nerves and the arterial vasculature and can be present from the skull base to the aortic arch (2). Approximately $90 \%$ of paragangliomas are benign. Furthermore, paragangliomas can arise in multiple locations, particularly in patients with succinate dehydrogenase subunit B, C, or D germline mutations (SDHB, SDHC, and SDHD, respectively) (2-4). In most cases, conventional imaging methods, such as CT and MRI, combined with arteriography can confirm the diagnosis of a paraganglioma. Nevertheless, radiologic data may not be sufficient to characterize lesions. Many tumors are not accessible for biopsy because of the risk of neurologic complications and the highly vascular nature of paragangliomas (5). Thus, other techniques may be helpful to confirm a diagnosis of paraganglioma and to disclose whether such tumors are multifocal.

As with most other neuroendocrine tumors, somatostatin receptor scintigraphy (SRS) can be used for visualization of these paragangliomas ( 6 ). In addition, these tumors are able to take up and decarboxylate amino acid precursors (7). In approximately $30 \%$ of all paragangliomas, the catecholamine pathway is active (8). In this pathway, the amino acid phenylalanine is metabolized to dopamine, adrenalin, and noradrenalin. As a noradrenalin analog, metaiodobenzylguanidine (MIBG), which is most commonly labeled with either ${ }^{123} \mathrm{I}$ or ${ }^{131} \mathrm{I}$, is a radiopharmaceutical that is taken up by the noradrenalin transporter. The combination of an active catecholamine pathway and the presence of uptake mechanisms for catecholamine metabolites enables the use of MIBG for visualizing paragangliomas (9).

The diagnostic yield of SRS and MIBG for the visualization of head and neck paragangliomas has been determined in several studies, but a direct comparison has been made in only few patients $(5,6,8-10)$, The present study was specif- 
ically initiated to compare the diagnostic sensitivity of SRS with MIBG scintigraphy in a group of patients with head and neck paragangliomas and also included an analysis of the performance of CT or MRI. These imaging results were compared with a composite reference standard, which consisted of clinical findings, morphologic imaging results, and histologic and follow-up data if available. The secondary aim of this study was to assess the relationship between catecholamine production of paragangliomas and tracer uptake, in the expectation that a better understanding of this relationship could improve the selection of patients for specific imaging studies.

\section{MATERIALS AND METHODS}

The study was performed in a university hospital setting, which served as a tertiary reference center for the northern part of The Netherlands. All patients included in this series were initially referred to the department of ear, nose, and throat diseases of the UMC Groningen. They were considered to have a head and neck paraganglioma on the basis of their own complaints and the localization and radiographic appearance of the tumor or had a histopathologically proven head and neck paraganglioma with a clinical indication for restaging. In most cases, part of this work-up had been completed at the referring hospital. All patients were referred to the outpatient endocrinology clinic for an additional work-up and underwent a diagnostic protocol including SRS, MIBG, and CT or MRI of the head and neck region, as well as biochemical analysis. This protocol was completed within 3 mo in all patients. Consecutive patients, who were evaluated between January 1993 and January 2007, were prospectively included in the present analysis, except for patients younger than $18 \mathrm{y}$ and those who were pregnant. Imaging methods were undertaken in random order. After completion of the work-up, results were discussed in multidisciplinary meetings and patients requiring surgery were operated on by ear, nose, and throat specialists.

\section{SRS}

Twenty-four hours after administration of $200 \mathrm{MBq}$ of ${ }^{111} \mathrm{In}$ octreotide (OctreoScan; Mallinckrodt), planar total-body scans (4 spot views of $10 \mathrm{~min}$ in a 128 matrix and side views of the head and neck) were obtained using a large-field-of-view double-head $\gamma$-camera (MULTISPECT 2; Siemens Inc.) with a medium-energy all-purpose collimator. System resolution was $12 \mathrm{~mm}$ in full width at half maximum at a $10-\mathrm{cm}$ distance. Both the 173- and the 247$\mathrm{keV}$ photopeaks of ${ }^{111} \mathrm{In}$ were used (15\% windows for each). In addition, in some patients SPECT acquisition and processing were performed by taking 64 projections $\left(2 \times 32 ; 5.6^{\circ}\right.$ for each 30 -s step in a $128 \times 128$ matrix). The total scan procedure took about 60 min. Transaxial tomograms were reconstructed without prefiltering with a Butterworth cutoff filter frequency of 0.35 . When necessary because of interfering bowel activity, additional 48-h images were recorded. This scanning protocol was performed according to Dutch guidelines (11).

\section{MIBG Imaging}

Fifteen minutes after administration of 10 drops of Lugol's solution (to prevent thyroid uptake of possibly formed free ${ }^{123} \mathrm{I}$ ), $200 \mathrm{MBq}$ of ${ }^{123} \mathrm{I}-\mathrm{MIBG}$ were administered. Twenty-four hours later, planar total-body scans ( 4 spot views of 10 min in a 256 matrix) were acquired using the same $\gamma$-camera as for the SRS scan. A medium- energy all-purpose collimator was used, and the $158-\mathrm{keV}$ photopeak was registered using a $15 \%$ window. In addition, a SPECT acquisition was done using the same specifications as described for SRS. Transaxial tomograms were reconstructed without prefiltering with a Butterworth cutoff filter frequency of 0.2-0.4, and attenuation correction was performed with a Chang's attenuation coefficient of $0.11 \mathrm{~cm}^{-1}$. Because of the interval of at least $1 \mathrm{wk}$ that elapsed between scans, no interference between the tracers was expected. This scanning protocol also followed Dutch guidelines (12).

\section{CT}

CT (1- to 16-slice scanner; Siemens Medical Systems) was performed before and during intravenous contrast enhancement using 3- to 10-mm-thick slices. The CT scan covered the head and neck area of all patients and any additional body region in which lesions were suspected. These CT scans were interpreted by dedicated specialists as part of routine patient care. At the time of data analysis, the investigators again reviewed the results, and in discrepant cases a consensus was reached after multidisciplinary discussion.

\section{MRI}

MRI was performed on a 1.5-T scanner (Vision; Siemens). The imaging protocol included standard spin-echo T1-weighted scans (repetition time [TR]/echo time [TE], 700/14), proton-density scans, and T2-weighted scans (TR/TE/second TE, 2,500/20/90) A spin-echo technique was used to obtain 5-mm contiguous 3-dimensional sections of the abdomen. T1-weighted images (TR/TE, 700/14) and T2-weighted images (TR/TE, 2,500/20/90) were obtained. T1-weighted images were also acquired after the intravenous administration of gadolinium-DTPA $(0.2 \mathrm{~mL} / \mathrm{kg}$ of body weight, Magnevist; Schering).

\section{Image Interpretation}

Because our diagnostic protocol was performed over a considerable period, improvements in diagnostic equipment arose for all modalities during the course of the study. However, basic detection principles did not change, and possible bias due to changes in equipment was assumed to be small. All SRS and MIBG scans were read twice. The first assessment was directly after the image reconstruction as part of patient care; the second was at the time of data analysis, and other imaging and biochemical information was masked. For both tracers, the number and location of lesions were scored. In addition, for each lesion, an uptake grade was assigned ( 0 , no uptake [a cold spot]; 1, lesion uptake = background uptake; 2 , lesion uptake $>$ background uptake; 3 , lesion uptake $\gg$ background uptake). Background was defined as uptake in normal neck musculature. In this way, a semiquantitative uptake score was established grading the amount of whole-body tumor uptake for both tracers. Lesions were considered positive for tumor if the uptake was at least 2 and could not be attributed to normal physiologic processes.

\section{Composite Reference}

As a composite reference standard for the presence of tumor lesions, we used all available cytologic, histologic, and imaging results, as well as follow-up data. We consider this approach to be optimal, because cytologic and histologic verification of all lesions is not feasible (13-16). Lesions were considered positive for tumor when imaging showed an abnormal mass, when clinical work-up produced a strong suspicion of paraganglioma (e.g., a visible tumor mass in the external auditory canal on otoscopy), or when histologic findings indicated paraganglioma. In many patients, histologic confirmation of all lesions was neither feasible nor justifiable. In cases of 
additional findings on SRS or MIBG imaging, these were verified with CT, MRI, ${ }^{18} \mathrm{~F}-3$,4-dihydroxyphenylalanine (DOPA) PET, bone scintigraphy, surgery, or follow-up whenever possible.

\section{Biochemical Measurements}

In each patient, three 24-h urine collections were obtained to document metanephrine and normetanephrine excretion. Biochemical results were classified as abnormal when the average value of these 3 measurements was elevated. Metanephrine levels of more than $99 \mu \mathrm{mol} / \mathrm{mol}$ of creatinine and metanephrine levels of more than $260 \mu \mathrm{mol} / \mathrm{mol}$ of creatinine were considered abnormal. Biochemical analysis was performed as described previously (17-19).

\section{Data and Statistical Analysis}

Analysis was performed at 2 levels. At the first level, individual patients were analyzed. Image findings were considered positive when at least 1 lesion in a patient was considered positive. At the second level, individual lesions were counted for all imaging modalities.

Sensitivities were calculated using the composite reference standard and were compared using paired observations and the McNemar test. Patient-based sensitivity was calculated as the number of patients with a positive test result (at least 1 lesion detected) divided by the total number of patients. For the comparison of number of lesions per region, the Wilcoxon rank test was used. For correlations between biochemical parameters and scan results, the Spearman correlation coefficient was calculated. A 2 -sided $P$ value of less than 0.05 was considered significant. The statistical tests were performed using the SPSS package, version 12.0.

\section{RESULTS}

\section{Patients}

Twenty-nine consecutive patients (17 women and 12 men; median age, 63 y [range, 33-94 y]) participated in this study

TABLE 1

Patient Characteristics

\begin{tabular}{|c|c|c|c|c|c|c|c|c|c|}
\hline $\begin{array}{c}\text { Patient } \\
\text { no. }\end{array}$ & $\begin{array}{l}\text { Age } \\
\text { (y) }\end{array}$ & Sex & Tumor type & SRS lesions & MIBG lesions & $\mathrm{CT} / \mathrm{MRI}$ & $\begin{array}{l}\text { Blood } \\
\text { pressure* }\end{array}$ & $\begin{array}{l}\text { Elevated urinary } \\
\text { catecholamine } \\
\text { metabolites }\end{array}$ & $\begin{array}{l}\text { Confirmation } \\
\text { or treatment }\end{array}$ \\
\hline 1 & 47 & $\mathrm{~F}$ & Glomus caroticum L & Neck L & Neck L & 0 & Normal & & Follow-up \\
\hline 2 & 55 & M & Glomus vagale L & Jaw L & Jaw L & Jaw L & Hypertension & & Surgery \\
\hline 3 & 40 & $\mathrm{~F}$ & $\begin{array}{l}\text { Glomus caroticum R + } \\
\text { L, pheochromocytoma } \\
\text { L, SDHD mutation }\end{array}$ & Jaw $R+L$ & $\begin{array}{l}\text { Left adrenal } \\
\text { gland }\end{array}$ & Jaw $R+L$ & Hypertension & Normetanephrine & Surgery \\
\hline 4 & 33 & M & Glomus tympanicum $\mathrm{R}$ & Ear $\mathrm{R}$ & 0 & Ear R & Normal & & Surgery \\
\hline 5 & 54 & $\mathrm{~F}$ & Glomus tympanicum L & 0 & 0 & Skull base $\mathrm{L}$ & Hypertension & & Surgery \\
\hline 6 & 51 & $M$ & Glomus temporale L & Ear L & 0 & Ear L & Hypertension & & Surgery \\
\hline 7 & 71 & M & Glomus caroticum L & Jaw $\mathrm{R}$ & Jaw $\mathrm{R}$ & Jaw $\mathrm{R}$ & Normal & & Surgery \\
\hline 8 & 39 & $\mathrm{~F}$ & Glomus tympanicum L & Ear L & 0 & Ear L & Normal & Metanephrine & Surgery \\
\hline 9 & 57 & $\mathrm{~F}$ & Glomus temporale L & Ear L & 0 & Ear L & Normal & & Surgery \\
\hline 10 & 52 & M & Glomus caroticum L & Neck L & 0 & Neck L & Hypertension & & Embolization \\
\hline 11 & 71 & $\mathrm{~F}$ & Glomus temporale L & Ear L & Ear L & Ear L & Normal & & Follow-up \\
\hline 12 & 94 & $\mathrm{~F}$ & Glomus tympanicum R & Ear $\mathrm{R}$ & 0 & Ear $\mathrm{R}$ & Normal & & Follow-up \\
\hline 13 & 65 & $\mathrm{~F}$ & $\begin{array}{l}\text { Glomus tympanicum R; } \\
\text { carcinoid R lung }\end{array}$ & $\begin{array}{l}\text { Ear } \mathrm{R} \text {, lung } \\
\text { hilus } \mathrm{R}\end{array}$ & 0 & Ear $\mathrm{R}$ & Normal & & $\begin{array}{l}\text { Follow-up, } \\
\text { surgery }\end{array}$ \\
\hline 14 & 70 & $\mathrm{~F}$ & $\begin{array}{l}\text { Glomus jugularis } L+R \text {, } \\
\text { caroticum } L\end{array}$ & $\begin{array}{c}\text { Neck } R+L \\
\text { mastoid } R\end{array}$ & Neck $R+L$ & $\begin{array}{r}\text { Neck } R+L, \\
\text { mastoid } R\end{array}$ & Hypertension & & $\begin{array}{l}\text { Follow up, } \\
\text { histology }\end{array}$ \\
\hline 15 & 68 & M & Glomus temporale $\mathrm{R}$ & Mastoid R & Mastoid R & Mastoid R & Normal & Metanephrine & Follow-up \\
\hline 16 & 80 & $\mathrm{~F}$ & Glomus tympanicum R & Ear $\mathrm{R}$ & 0 & Ear $\mathrm{R}$ & Normal & & Surgery \\
\hline 17 & 75 & $\mathrm{~F}$ & Glomus temporale L & $\begin{array}{l}\text { Skull base, } \\
\text { neck } R+L\end{array}$ & $\begin{array}{l}\text { Skull base, } \\
\text { neck } R+L\end{array}$ & Skull base & Hypertension & Metanephrine & Follow-up \\
\hline 18 & 71 & M & Glomus temporale L & Ear L & 0 & Ear L & Normal & Metanephrine & Follow-up \\
\hline 19 & 33 & $M$ & Glomus jugularis L & Skull base L & 0 & Skull base L & Hypertension & & Surgery \\
\hline 20 & 47 & $\mathrm{~F}$ & Glomus jugularis $\mathrm{R}$ & Skull base $\mathrm{R}$ & 0 & Skull base $\mathrm{R}$ & Hypertension & & Surgery \\
\hline 21 & 55 & M & Glomus vagale $L$ & Skull base $\mathrm{L}$ & 0 & Skull base $\mathrm{L}$ & Normal & & Surgery \\
\hline 22 & 68 & $M$ & Glomus vagale L & Skull base L & Skull base L & Skull base L & Normal & & Surgery \\
\hline 23 & 61 & $M$ & Glomus jugularis $\mathrm{R}$ & Ear $\mathrm{R}$ & Ear R & Ear $\mathrm{R}$ & Normal & & Follow-up \\
\hline 24 & 53 & M & Glomus caroticum L & Neck L & Neck L & Neck L & Hypertension & & Embolization \\
\hline 25 & 63 & $\mathrm{~F}$ & Glomus jugularis L & Ear L & 0 & Ear L & Normal & & Follow-up \\
\hline 26 & 65 & $\mathrm{~F}$ & $\begin{array}{l}\text { Glomus caroticum } \\
\mathrm{L}+\mathrm{R} \text {; abdominal } \\
\text { carcinoid }\end{array}$ & $\begin{array}{l}\text { Neck L; } \\
\text { abdomen R }\end{array}$ & $\begin{array}{l}\text { Neck L; } \\
\text { abdomen R }\end{array}$ & Neck L $+R$ & Normal & & Surgery \\
\hline 27 & 64 & $\mathrm{~F}$ & Glomus caroticum R & Jaw $R$ & Jaw $R$ & Jaw $R$ & Normal & & Follow-up \\
\hline 28 & 71 & $\mathrm{~F}$ & Glomus caroticum R & Jaw $R$ & 0 & Jaw $R$ & Normal & & Follow-up \\
\hline 29 & 65 & M & Glomus tympanicum $\mathrm{R}$ & 0 & 0 & Ear $\mathrm{R}$ & Normal & & Follow-up \\
\hline
\end{tabular}


TABLE 2

Patient- and Lesion-Based Analysis of CT or MRI, SRS, and MIBG of Head and Neck Paraganglioma in 29 Patients

\begin{tabular}{lccr}
\hline \multicolumn{1}{c}{ Analysis } & CT or MRI & SRS & MIBG \\
\hline Patient-based & & & \\
Positive patients $(n)$ & $27^{\star}$ & $27^{\star}$ & 13 \\
Sensitivity (\%) & 93 & 93 & 44 \\
95\% Cl (\%) & $77-98$ & $77-98$ & $23-61$ \\
Lesions ( $n)$ & & & \\
$\quad$ Mean & 1.07 & 1.17 & 0.52 \\
Range & $0-3$ & $0-3$ & $0-3$ \\
Lesion-based & & & \\
Positive lesions (n) & $31^{\dagger}$ & $34^{\dagger}$ & 15 \\
Sensitivity (\%) & 82 & 89 & 42 \\
95\% Cl (\%) & $65-92$ & $75-97$ & $26-59$
\end{tabular}

${ }^{*}$ SRS and CT were significantly better than MIBG $(P<0.001)$.

${ }^{\dagger} \mathrm{SRS}$ and CT were better than MIBG $(P=0.001)$.

All sensitivities are based on comparison with composite reference standard.

(Table 1). All were ultimately considered to have a paraganglioma. This diagnosis was based on surgical or histologic findings in 16 patients and on follow-up findings in 11 patients. In 2 patients, the morphologic appearance of the lesion indicated paraganglioma, and they were treated with embolization. The median time between SRS and MIBG scintigraphy was $7 \mathrm{~d}$, and the median time between SRS/MIBG and CT or MRI was $78 \mathrm{~d}$. Conventional imaging was performed on all patients: either CT of the head and neck region (14 patients) or MRI of the head and neck region (15 patients).

\section{Patient-Based Analysis}

All 29 patients were considered positive for paragangliomas, on the basis of the composite reference standard (Table
2). Morphologic imaging and SRS correctly identified 27 of 29 patients (sensitivity, 93\%; 95 confidence interval [CI], 77\%-98\%), whereas MIBG correctly identified 13 patients (sensitivity, 44\%; 95 CI, 23\%-61\%). SRS detected significantly more tumor-positive patients than did MIBG $(P<$ 0.001). We found that SRS uptake was more intense than MIBG uptake in 7 patients, was equal to MIBG uptake in 3 patients, and was less intense than MIBG uptake in 2 patients.

\section{Lesion-Based Analysis}

Thirty-eight lesions were identified using the composite reference standard. Morphologic imaging correctly detected 31 of these lesions (sensitivity, 82\%; 95\% CI, 65\%-92\%; 1.07 lesions per patient), SRS detected 34 (sensitivity, 89\%; 95\% CI, 75\%-98\%; 1.17 lesions per patient), and MIBG detected 15 (sensitivity, 42\%; 95\% CI, 26\%-59\%; 0.52 lesions per patient). SRS detected more lesions than did MIBG ( $P=0.0001$; an example is shown in Fig. 1). In 6 patients, more than one lesion was present in the head and neck region (Table 1 ).

\section{Secondary Tumors and Discrepant Cases}

In 3 patients (10\%), previously unknown neuroendocrine tumors were found outside the head and neck region. In 1 patient, SRS showed a pulmonary lesion that was due to a carcinoid (patient 13). This patient was negative for SDHB, SDHD, and RET protooncogene mutations. In another patient (patient 26), SRS detected a lesion in the abdomen (Fig. 2 ). This patient was found to have an SDHB mutation. This patient refused surgery, but a carcinoid was clinically suspected. These lesions did not show MIBG uptake. However, MIBG scintigraphy led to the detection of a left-sided adrenal pheochromocytoma, which was not detected on SRS (patient 3). This patient was found to have an SDHD gene mutation.
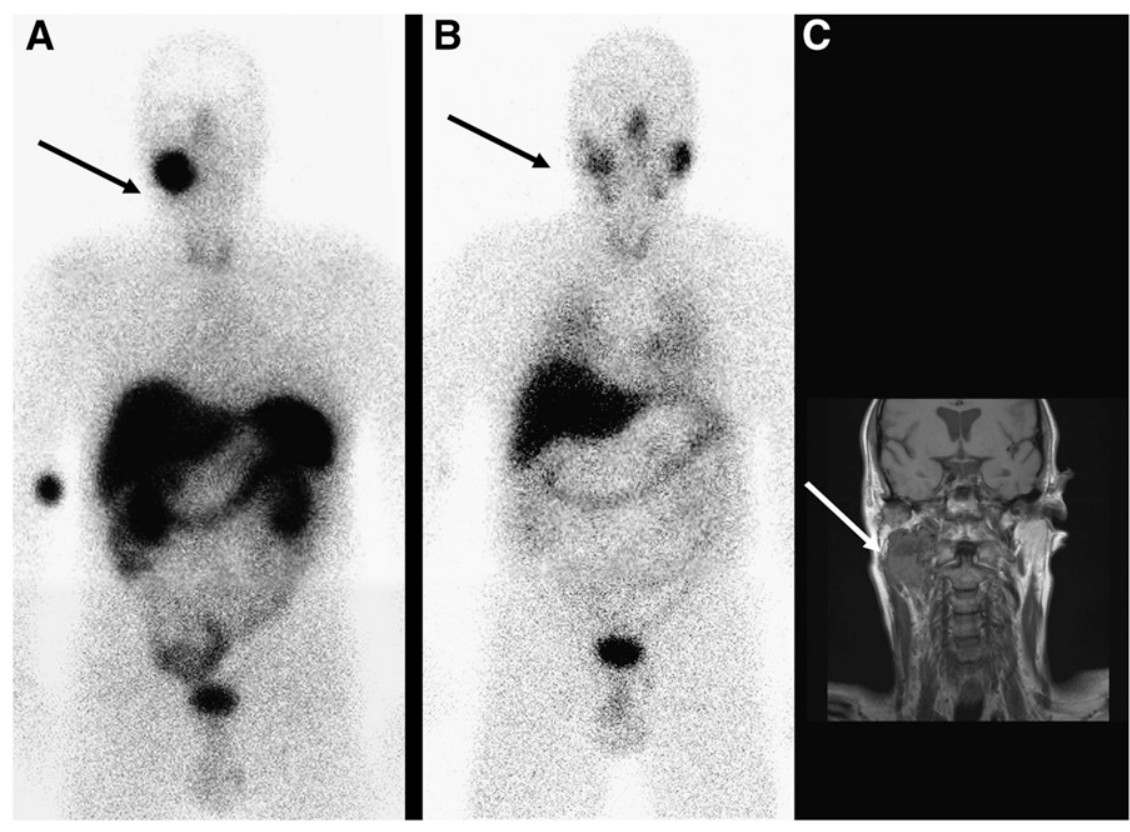

FIGURE 1. MIBG-negative paraganglioma. (A and $B$ ) SRS image (A) shows pathologic uptake (grade 3 ) in right jaw, whereas on MIBG scan (B) this uptake (grade 1) in not easily identified. (C) MRI coronal slice shows lesion in right jaw. 
A

FIGURE 2. Additional abdominal lesion detected with SRS. (A) SRS image shows uptake in left neck (red arrow, grade 3) and in abdomen, suspected to be due to carcinoid tumor (blue arrow, grade 3 ). (B) On MIBG scan, neck lesion appears much smaller (red arrow, grade 2) and abdominal lesion shows no uptake (grade 1). (C) SRS and MRI fusion image shows neck lesion.

\section{Biochemical Results}

Four patients had elevated urinary metanephrine excretion, whereas 1 patient (the patient with adrenal pheochromocytoma) had elevated urinary normetanephrine excretion (Table 1). Elevated levels of urinary meta- or normetanephrine excretion were correlated with the number of SRS lesions per patient $(r=0.51, P=0.005)$ and the number of MIBG lesions ( $r=0.47, P=0.02$ ). These relationships were not significant on a patient-based analysis (data not shown). The correlation between the presence of hypertension and abnormal urinary metanephrine or normetanephrine excretion was not significant (data not shown).

\section{DISCUSSION}

In our cohort of 29 consecutive patients with head and neck paraganglioma, it was evident that SRS was superior to MIBG imaging in the detection of head and neck paragangliomas. SRS performed as well as morphologic imaging for the detection of head and neck paraganglioma in a patientbased analysis and detected some additional lesions, not found with the morphologic methods, in the head and neck region. Thus, SRS is likely to contribute to optimal lesion characterization in this patient category. Furthermore, in view of the low yield of MIBG imaging, we suggest that this scan should not be used routinely in the evaluation of head and neck paraganglioma.

The main difference between the present report and previous studies on the performance characteristics of SRS and MIBG imaging is that most other studies also included paragangliomas outside the head and neck region $(5,8,10)$. Importantly, our cohort comprised only patients who were considered to have head and neck paraganglioma and who had been referred from the ear, nose, and throat department for protocolized imaging studies. Thus, our study does not allow conclusions to be drawn on diagnostic yield and the specificity of SRS and MIBG when a diagnosis of head and
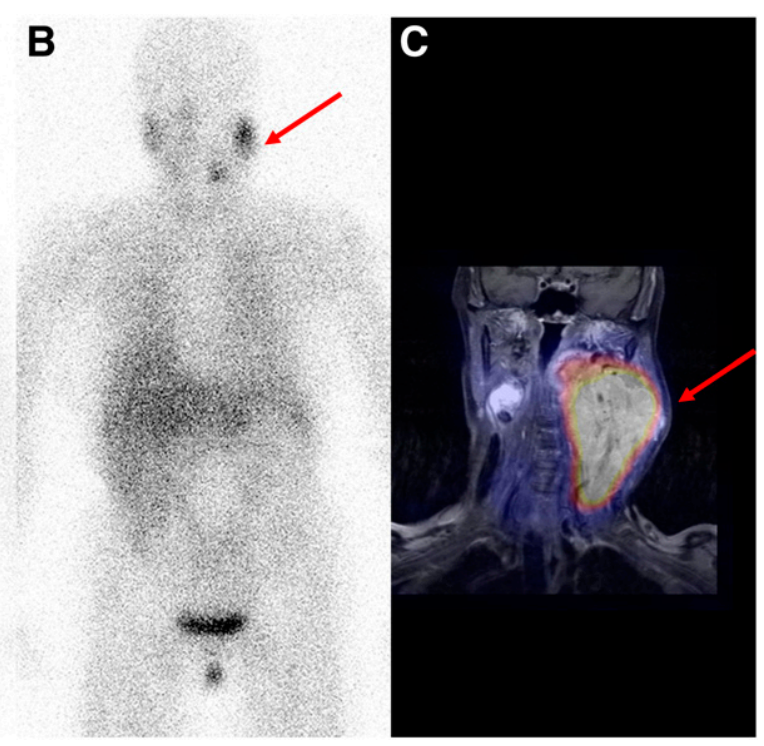

neck paraganglioma is uncertain. Nonetheless, the present results with respect to the comparison between SRS and MIBG are in line with those of other studies. In a study comprising 8 patients, Muros et al. showed that SRS had a sensitivity of $100 \%$, compared with $56 \%$ for MIBG (6). In a large retrospective study (8), the clinical records of 236 patients with benign paraganglioma, including those with tumors outside the head and neck region, were evaluated. In 25 of these patients, on whom MIBG imaging was performed, this technique could detect only $62 \%$ of the paragangliomas, but no comparison could be made with SRS. In a study comprising 34 patients, Kwekkeboom et al. (10) demonstrated a sensitivity of $94 \%$ with SRS for the detection of paragangliomas. Importantly, that study showed that the use of whole-body imaging revealed additional paraganglioma lesions in 9 patients.

A potentially important clinical finding of our study is that SRS resulted in the detection of a previously unknown carcinoid tumor in one patient and brought about clinical suspicion in another. To our knowledge, the coincidence of head and neck paraganglioma with carcinoid tumors has not been reported. However, the only coexisting adrenal pheochromocytoma in the present series was discovered with MIBG but not with SRS. This finding agrees with a report that showed adrenal pheochromocytoma in 7 of 40 patients with either an SDHD mutation or a positive family history for head and neck paragangliomas (20). Thus, there remains an indication to perform MIBG imaging when (adrenal) pheochromocytoma is clinically suspected. In our study, 5 (17\%) of 29 subjects, including the patient with adrenal pheochromocytoma, had elevated urinary metanephrine or normetanephrine excretion. In comparison, in the retrospective analysis of 236 benign paragangliomas, excess catecholamine production was found in $31 \%$ of tested subjects (8). In the current report, there was only a modest correlation of abnormal catecholamine production, as documented by elevated urinary metanephrines or normetanephrine excretion, 
with SRS as well as with MIBG results on a lesion-based analysis. However, because urinary catecholamine measurements can easily be obtained, we propose that MIBG be added to SRS in patients with clinically or biochemically suspected (adrenal) pheochromocytoma.

Recently, other imaging techniques have been used to detect neuroendocrine tumors. Preliminary data have shown a high yield of ${ }^{18} \mathrm{~F}$-DOPA PET in carcinoid tumors (16). Thus far, ${ }^{18} \mathrm{~F}$-DOPA has been used in only small series of patients with paraganglioma, but the results are promising (21-23). Although ${ }^{18} \mathrm{~F}$-DOPA PET was able to detect more paraganglioma lesions than were MRI and MIBG, no direct comparison with SRS has been made. Of note, the performance of ${ }^{18} \mathrm{~F}$-DOPA PET may be less optimal in paragangliomas that result from SDH gene mutations, because these tumors are prone to dedifferentiation. In such cases, the use of ${ }^{18} \mathrm{~F}-$ DOPA PET, $6-{ }^{18} \mathrm{~F}$-fluorodopamine PET, or FDG PET may be useful $(3,22,23)$.

Finally, we did not present follow-up data from repeated nuclear imaging studies, but it seems reasonable to assume that SRS is of merit for detecting residual or recurrent paraganglioma after initial treatment and that ${ }^{177} \mathrm{Lu}-$ tetraazacyclododecanetetraacetic acid-Tyr ${ }^{3}$-octreotate may be useful for treating residual disease in selected patients (24).

\section{CONCLUSION}

We have documented a high sensitivity of SRS for the detection of paragangliomas in the head and neck region-a sensitivity equal to that of morphologic imaging methods. However, SRS has 2 major theoretic advantages that should be confirmed in further studies: its ability to characterize lesions as being of neuroendocrine origin and its ability to image the whole body, which can lead to the detection of additional neuroendocrine lesions.

\section{ACKNOWLEDGMENTS}

One of the authors is financially supported by grant 2003-2936 from the Dutch Cancer Foundation. We appreciate the long-standing cooperation of our highly respected coinvestigator, Professor Frans Albers, who unexpectedly passed away during the production of this paper.

\section{REFERENCES}

1. Lee JH, Barich F, Karnell LH, et al. National Cancer Data Base report on malignant paragangliomas of the head and neck. Cancer. 2002;94:730-737.

2. Baysal BE, Willett-Brozick JE, Lawrence EC, et al. Prevalence of SDHB, SDHC, and SDHD germline mutations in clinic patients with head and neck paragangliomas. J Med Genet. 2002;39:178-183.

3. Timmers HJ, Kozupa A, Chen CC, et al. Superiority of fluorodeoxyglucose positron emission tomography to other functional imaging techniques in the evaluation of metastatic SDHB-associated pheochromocytoma and paraganglioma. J Clin Oncol. 2007;25:2262-2269.

4. McCaffrey TV, Myssiorek D, Marrinan M. Head and neck paragangliomas: physiology and biochemistry. Otolaryngol Clin North Am. 2001;34:837-844.

5. Duet M, Sauvaget E, Petelle B, et al. Clinical impact of somatostatin receptor scintigraphy in the management of paragangliomas of the head and neck. J Nucl Med. 2003;44:1767-1774.

6. Muros MA, Llamas-Elvira JM, Rodriguez A, et al. ${ }^{111}$ In-pentetreotide scintigraphy is superior to ${ }^{123} \mathrm{I}-\mathrm{MIBG}$ scintigraphy in the diagnosis and location of chemodectoma. Nucl Med Commun. 1998;19:735-742.

7. Pearse AG. The APUD cell concept and its implications in pathology. Pathol Аnпu. 1974;9:27-41.

8. Erickson D, Kudva YC, Ebersold MJ, et al. Benign paragangliomas: clinical presentation and treatment outcomes in 236 patients. J Clin Endocrinol Metab. 2001;86:5210-5216.

9. Maurea S, Cuocolo A, Reynolds JC, et al. Iodine-131-metaiodobenzylguanidine scintigraphy in preoperative and postoperative evaluation of paragangliomas: comparison with CT and MRI. J Nucl Med. 1993;34:173-179.

10. Kwekkeboom DJ, van Urk H, Pauw BK, et al. Octreotide scintigraphy for the detection of paragangliomas. J Nucl Med. 1993;34:873-878.

11. Somatostatine-receptor-scintigrafie. In: Urk P, Barneveld PC, eds. Aanbevelingen Nucleaire Geneeskunde. Neer, The Netherlands: Kloosterhof Acquisitie ServicesUitgeverij; 2007:64-67.

12. MIBG-scintigrafie. In: Urk P, Barneveld PC, eds. Aanbevelingen Nucleaire Geneeskunde. Neer, The Netherlands: Kloosterhof Acquisitie ServicesUitgeverij; 2007:60-63.

13. Strobel K, Skalsky J, Kalff V, et al. Tumour assessment in advanced melanoma: value of FDG-PET/CT in patients with elevated serum S-100B. Eur J Nucl Med Mol Imaging. 2007;34:1366-1375.

14 Koopmans KP, Neels OC, Kema IP, et al. Improved staging of patients with carcinoid and islet cell tumors with ${ }^{18} \mathrm{~F}$-dihydroxy-phenyl-alanine and ${ }^{11} \mathrm{C}-5$ hydroxy-tryptophan positron emission tomography. J Clin Oncol. 2008 20;26: 1489-1495.

15. Koopmans KP, de Groot JW, Plukker JT, et al. ${ }^{18}$ F-dihydroxyphenylalanine PET in patients with biochemical evidence of medullary thyroid cancer: relation to tumor differentiation. J Nucl Med. 2008;49:524-531.

16. Koopmans KP, de Vries EG, Kema IP, et al. Staging of carcinoid tumours with ${ }^{18}$ F-DOPA PET: a prospective, diagnostic accuracy study. Lancet Oncol. 2006; 7:728-734.

17. Kema IP, Meiborg G, Nagel GT, Stob GJ, Muskiet FA. Isotope dilution ammonia chemical ionization mass fragmentographic analysis of urinary 3Omethylated catecholamine metabolites: rapid sample clean-up by derivatization and extraction of lyophilic samples. J Chromatogr Biomed Appl. 1993;671: $181-189$.

18. Kema IP, de Vries EG, Slooff MJ, Biesma B, Muskiet FA. Serotonin, catecholamines, histamine, and their metabolites in urine, platelets, and tumor tissue of patients with carcinoid tumors. Clin Chem. 1994;40:86-95.

19. Jager PL, Meijer WG, Kema IP, et al. L-3-[ $\left.{ }^{123} I\right]$ iodo-alpha-methyltyrosine scintigraphy in carcinoid tumors: correlation with biochemical activity and comparison with [ ${ }^{111}$ In-DTPA-D-Phe1]-octreotide imaging. J Nucl Med. 2000;41: 1793-1800.

20. van Houtum WH, Corssmit EP, Douwes Dekker PB, et al. Increased prevalence of catecholamine excess and phaeochromocytomas in a well-defined Dutch population with SDHD-linked head and neck paragangliomas. Eur J Endocrinol. 2005;152:87-94.

21. Brink I, Schaefer O, Walz M, Neumann HP. Fluorine-18 DOPA PET imaging of paraganglioma syndrome. Clin Nucl Med. 2006;31:39-41.

22. Hoegerle S, Ghanem N, Altehoefer C, et al. ${ }^{18} \mathrm{~F}$-DOPA positron emission tomography for the detection of glomus tumours. Eur J Nucl Med Mol Imaging. 2003;30:689-694.

23. Ilias I, Yu J, Carrasquillo JA, et al. Superiority of $6-\left[{ }^{18} \mathrm{~F}\right]$-fluorodopamine positron emission tomography versus $\left[{ }^{131} \mathrm{I}\right]$-metaiodobenzylguanidine scintigraphy in the localization of metastatic pheochromocytoma. J Clin Endocrinol Metab. 2003;88:4083-4087.

24. van Essen M, Krenning EP, Kooij PP, et al. Effects of therapy with $\left[{ }^{177} \mathrm{Lu}-\right.$ DOTA0, Tyr3] octreotate in patients with paraganglioma, meningioma, small cell lung carcinoma, and melanoma. J Nucl Med. 2006;47:1599-1606. 